\title{
PROCESSO DE SELEÇÃO DE MECANISMOS PARA COMPARTILHAMENTO DO CONHECIMENTO: O CASO DE UMA INDÚSTRIA BRASILEIRA DO RAMO DE PNEUS E BORRACHAS
}

\section{SELECTION PROCESS OF KNOWLEDGE SHARING MECHANISMS: A BRAZILIAN TIRES AND RUBBER ARTIFACTS INDUSTRY CASE}

\author{
Suelen Cavalli \\ Pontifícia Universidade Católica do Rio Grande do Sul - PUC-RS \\ suelenc10@gmail.com
}

Mírian Oliveira

Pontifícia Universidade Católica do Rio Grande do Sul - PUC-RS

$\underline{\text { miriano@pucrs.br }}$

Andrea Raymundo Balle

Pontifícia Universidade Católica do Rio Grande do Sul - PUC-RS arballe@gmail.com

Submissão: $11 / 04 / 2018$

Aprovação: 08/08/2018

\begin{abstract}
RESUMO
As organizações têm dificuldade de reter o conhecimento individual para que se tornem o conhecimento organizacional, e por isso se faz necessário um processo cuidadoso para selecionar mecanismos que devem ser adotados no compartilhamento de conhecimento. Esta pesquisa aborda o processo de seleção de mecanismos para o compartilhamento do conhecimento nas áreas administrativas corporativas de uma indústria brasileira de artefatos de borrachas. Caracteriza-se um estudo de caso único, do tipo exploratório. A coleta de dados ocorreu através de entrevistas semiestruturadas e observação direta, analisadas com a técnica análise de conteúdo. Os resultados obtidos demonstram quais mecanismos de compartilhamento do conhecimento são identificados na organização e se eles são eficazes, além de revelar que há necessidade de implantar uma estratégia de gestão do conhecimento, e sugere os mecanismos: sistemas especialistas, páginas amarelas e incentivo ao mentoring. Este estudo contribui academicamente para a gestão do conhecimento.
\end{abstract}

Palavras-chave: Gestão do conhecimento. Compartilhamento do conhecimento. Indústria de artefatos de borracha. Mecanismos de compartilhamento do conhecimento. 


\begin{abstract}
Organizations have difficulty retaining individual knowledge to transform it in organizational knowledge, therefore a careful process to select the adopted mechanisms for knowledge sharing is necessary. This research approaches the process of selecting mechanisms for knowledge sharing in the corporate administrative areas of a Brazilian rubber artifact industry. It is characterized as an exploratory single case study. Data collection occurred through semistructured interviews and direct observation, analyzed with content analysis technique. Results show which knowledge sharing mechanisms are identified in the organization and if these mechanisms are effective, reveals the need to implement a knowledge management strategy, and suggests the following mechanisms: expert systems, yellow pages and incentive to mentoring. This study contributes academically to knowledge management.
\end{abstract}

Keywords: Knowledge management. Knowledge sharing. Rubber artifacts industry. Knowledge sharing mechanisms. 


\section{INTRODUÇÃO}

O conhecimento contido na empresa é de fundamental importância, visto que com o seu uso é possível melhorar os processos e fazer repensar os métodos pelos quais as empresas buscam cumprir sua missão. Conhecimento é uma mistura complexa de experiências, valores e informações (DAVENPORT; PRUSAK, 1998). Benbya e Belbaly (2005) dizem que as atividades realizadas para gerenciar o conhecimento devem ser integradas nos processos do negócio diariamente, para que seja possível desenvolver a memória organizacional, e facilitar a aprendizagem, além de assegurar a melhoria contínua. A importância do gerenciamento do conhecimento é tanta, e impacta tão diretamente nos resultados da qualidade das organizações, que a ABNT NBR ISO 9001:2015 ganhou um item específico sobre o conhecimento organizacional, desafiando muitos sistemas de gestão da qualidade (ASSOCIAÇÃO BRASILEIRA DE NORMAS TÉCNICAS, 2015). A gestão do conhecimento pode contribuir para as organizações serem mais inovadoras e atenderem as demandas dos clientes (HASHEMI; KHADIVAR; SHAMIZANJANI, 2018).

O conhecimento organizacional é proveniente do capital intelectual de cada indivíduo, sendo importante que a alta gestão incentive o melhor aproveitamento do conhecimento dos indivíduos (Jorge e Faléco, 2016). Compartilhar conhecimento, permitindo acesso a quem o necessita, é fundamental para as organizações, em especial para aquelas que operam em um ambiente globalizado (SOMANTRI et al., 2018). Para isto, os autores informam que podem ser adotados mecanismos de compartilhamento. Os mecanismos adotados para o compartilhamento do conhecimento (CC) devem buscar o envolvimento e a participação dos colaboradores no processo. Podem ser citados alguns mecanismos, como: lições aprendidas, mentoring, Wiki, entre outros (OLIVEIRA; MAÇADA; CURADO, 2014). Os mecanismos baseados em tecnologia facilitam os processos de gestão do conhecimento (HASHEMI; KHADIVAR; SHAMIZANJANI, 2018). No entanto, a seleção apropriada de ferramentas que podem auxiliar nos processos de gestão do conhecimento é um desafio para as organizações HASHEMI; KHADIVAR; SHAMIZANJANI, 2018).

O ano de 2015 iniciou com muitas dificuldades políticas que impactaram a economia, manifestações públicas e investigações no âmbito da Polícia Federal, denúncias na justiça, entre outros fatores que agravaram a situação do país. No setor da indústria de borrachas houve retração de quase $10 \%$ em 2015, sendo este o segundo ano consecutivo de queda na produção no Brasil (SINBORSUL, 2016). Os impactos negativos no mercado de trabalho, iniciados no ano de 2015, geraram o fechamento de cerca de 680 mil vagas de emprego no Brasil até agosto do ano de 2016, número que representa $1,4 \%$ da força de trabalho total, considerando-se apenas o mercado formal (SILVA, 2016). Pesquisa na mesma fonte revela que a indústria em geral foi um dos setores mais afetados, tendo em vista a destruição líquida de empregos que representa $6 \%$ das vagas durante o período de 12 meses, encerrado em agosto do ano de 2016.

Diante deste contexto, este trabalho tem como objetivo selecionar mecanismos para o $\mathrm{CC}$ nas áreas administrativas corporativas de uma indústria de artefatos de borrachas e pneumáticos brasileira. Além desta introdução, este trabalho apresenta uma revisão de literatura sobre mecanismos para compartilhamento de conhecimento (seção 2), os aspectos metodológicos adotados na pesquisa (seção 3), a análise do conteúdo (seção 4), a discussão dos resultados (seção 5), e a conclusão (seção 6).

\section{MECANISMOS PARA COMPARTILHAMENTO DO CONHECIMENTO}

Este trabalho possui a revisão da literatura dividida em conceitos (subseção 2.1), mecanismos para CC (subseção 2.2) e seleção de mecanismos (subseção 2.3). 


\subsection{CONCEITOS ASSOCIADOS AO COMPARTILHAMENTO DO CONHECIMENTO}

Os processos de gestão do conhecimento (GC) podem ser identificados como: criação, captura, refinamento, armazenamento, gerenciamento e disseminação do conhecimento (BOSE, 2004). O compartilhamento do conhecimento é considerado o processo mais importante de GC (GUINEA; WEBSTER; STAPLES, 2012). Desta forma, o foco deste trabalho está na disseminação do conhecimento, que de acordo com Bose (2004), é a etapa da GC que se preocupa em disponibilizar o conhecimento para qualquer pessoa que o necessite, independentemente do local em que se encontre, podendo, ou não, utilizar tecnologias.

Para abordar os mecanismos existentes para o CC, é necessário o entendimento sobre as interações do conhecimento tácito com o explícito e suas formas de conversão. Nonaka e Takeuchi (2008) afirmam que o conhecimento explícito é aquele que se pode expressar em formato de som, dados, manuais ou até mesmo palavras. Já o conhecimento tácito, para os autores, se caracteriza pela forma pessoal, o chamado know-how do indivíduo, que envolve percepções, e modelos mentais, difíceis de formalizar e compartilhar. De acordo com eles, o conhecimento tácito e o explícito interagem criando quatro formatos de conversão do conhecimento: socialização (conhecimento tácito para tácito), externalização (conhecimento tácito para explícito), combinação (conhecimento explícito para explícito) e internalização (conhecimento explícito para tácito).

Hansen, Nohria e Tierney (1999) informam que existem duas estratégias utilizadas pelas empresas que investem na GC, sendo elas denominadas codificação e personalização. Segundo os autores, a codificação é a transferência do conhecimento tácito para o explícito, sendo que estes poderão ficar armazenados, por exemplo, em um banco de dados para que possam ser localizados e utilizados facilmente por outras pessoas. Os mesmos autores informam que a personalização trata de um formato diferente de transferência de conhecimento, visto que procura compartilhá-lo estimulando o contato direto entre as pessoas, como diálogos individuais, telefone, videoconferências, entre outros. Hansen, Nohria e Tierney (1999) dizem que as empresas eficazes podem até utilizar as duas estratégias, porém, devem priorizar uma e utilizar a outra como apoio.

A partir da definição da estratégia a ser adotada pela organização, é que se definem quais serão os mecanismos para CC que melhor se adaptem à realidade em questão, podendo ser tecnológicos ou não. A criação de uma estrutura formal para CC é muito importante para impulsionar a empresa na transformação de processos internos orientados ao desenvolvimento de novas tecnologias (CAMPOS; FERREIRA; SILVA, 2009).

\subsection{TIPOS DE MECANISMOS PARA COMPARTILHAMENTO DO CONHECIMENTO}

Os mecanismos de CC podem, ou não, utilizar tecnologias da informação para ajudar a compartilhar o conhecimento (OLIVEIRA; MAÇADA; CURADO, 2014).

\subsubsection{Mecanismos não tecnológicos}

O Quadro 1 apresenta um resumo dos mecanismos não tecnológicos associados aos autores que mencionam os mesmos.

\begin{tabular}{|l|l|}
\hline \multicolumn{1}{|c|}{$\begin{array}{c}\text { Mecanismos Não } \\
\text { Tecnológicos }\end{array}$} & \multicolumn{1}{c|}{ Autores } \\
\hline $\begin{array}{l}\text { Comunidades de } \\
\text { práticas }\end{array}$ & $\begin{array}{l}\text { Chen } \text { et al., 2018; Binney, 2001; Bechky, 2003; Kankanhalli } \text { et } \\
\text { al.,2003; Benbya e Belbaly, 2005; O'Sullivan, 2007; Saito; } \\
\text { Umemoto; Ikeda, 2007. }\end{array}$ \\
\hline Contar Histórias & Bechky, 2003. \\
\hline
\end{tabular}




\begin{tabular}{|l|l|}
\hline Conversas Informais & $\begin{array}{l}\text { Hansen; Nohria; Tierney,1999; Kankanhalli } \text { et al., 2003; } \\
\text { Jasimuddin, 2007. }\end{array}$ \\
\hline Reuniões Face a Face & $\begin{array}{l}\text { Chen } \text { et al., 2018; Hansen; Nohria; Tierney,1999; Kankanhalli } \text { et } \\
\text { al., 2003; Jasimuddin, 2007. }\end{array}$ \\
\hline Mobilidade de Pessoal & Hansen; Nohria; Tierney,1999; Kankanhalli et al., 2003. \\
\hline Brainstorming & Kankanhalli et al., 2003; Hansen; Nohria; Tierney,1999. \\
\hline Lições Aprendidas & $\begin{array}{l}\text { Veronese, 2014; Benbya e Belbaly, 2005; Binney, 2001; Saito; } \\
\text { Umemoto; Ikeda, 2007; Bollinger e Smith, 2001. }\end{array}$ \\
\hline Mentoring & $\begin{array}{l}\text { Curtis; Taylor, 2018; Lawrence, 2008; Hansen; Nohria; } \\
\text { Tierney,1999; Bollinger e Smith, 2001. }\end{array}$ \\
\hline Melhores Práticas & $\begin{array}{l}\text { Benbya e Belbaly, 2005; Binney, 2001; Saito; Umemoto; Ikeda, } \\
\text { 2007; Bollinger e Smith, 2001. }\end{array}$ \\
\hline Trabalho em Equipe & Bollinger e Smith, 2001; Saito; Umemoto; Ikeda, 2007. \\
\hline Treinamentos & $\begin{array}{l}\text { Chen } \text { et al., 2018; Binney, 2001; Saito; Umemoto; Ikeda, 2007; } \\
\text { Hansen; Nohria; Tierney,1999; Kankanhalli } \text { et al., 2003. }\end{array}$ \\
\hline
\end{tabular}

Quadro 1 - Mecanismos não tecnológicos para o compartilhamento do conhecimento. Fonte: Adaptado de Oliveira, Maçada e Curado (2014).

Pode-se iniciar a abordagem de mecanismos não tecnológicos com o conceito de comunidades de prática, que se caracteriza por ser o meio pelo qual se pode desenvolver o conhecimento tácito, unindo as pessoas para que possam compartilhar ideias e aprender umas com as outras através das experiências compartilhadas (BINNEY, 2001). De acordo com Bechky (2003) os colaboradores podem participar das comunidades de práticas por meio de narrativas de histórias que estimulem o aprendizado e que proporcionem entendimentos do seu mundo através da intepretação de eventos organizacionais.

Conversas informais e reuniões face a face também se caracterizam como mecanismos não tecnológicos (HANSEN; NOHRIA; TIERNEY, 1999). A mesma fonte afirma que construir redes de pessoas para estimular o CC é importante, e pode ser realizada por meio da mobilidade de pessoal, que é a transferência de pessoas entre escritórios, reforçando os relacionamentos e objetivando uma cultura de prontidão de retorno às demandas de colegas.

Também pode ser citado como um mecanismo de CC não tecnológico as sessões de brainstorming, que são reuniões com o objetivo de trocar experiências e promover novos conceitos (KANKANHALLI et al., 2003), e as lições aprendidas, mecanismo pelo qual os indivíduos transferem o conhecimento que adquiriram em um projeto, para gerar valor ao negócio, aprimorar os processos e até reduzir custos (VERONESE, 2014). A busca por ferramentas que identifiquem a melhor maneira de fazer algo dentro de uma organização é denominada melhores práticas, e também pode ser considerada como um mecanismo não tecnológico voltado para a GC (BENBYA; BELBALY, 2005).

Para Lawrence (2008), mentoring se caracteriza por sessões onde um indivíduo com mais experiência pode discutir oportunidades e dificuldades com um indivíduo com menos experiência, objetivando o desenvolvimento de novas habilidades do indivíduo com menos experiência. Para o autor, o mentoring beneficia a organização, que obtém maior retenção, carreiras traçadas mais claramente, maiores competências de liderança, além de líderes prontos na hora certa. Já Bollinger e Smith (2001) destacam o trabalho em equipe como um importante mecanismo de interação, que promove a inovação, a cooperação entre os membros da equipe, e que prospera em uma cultura organizacional forte e de apoio à aprendizagem. Binney (2001) relata que é importante o desenvolvimento planejado do conhecimento tácito para que haja intervenções de formação, sejam elas com atribuições experienciais ou 
pertencentes a comunidades de interesse. $\mathrm{O}$ autor também afirma que além do treinamento tradicional, deve-se dar ênfase ao desenvolvimento de organizações de aprendizagem e habilidades colaborativas. Desta forma, pode-se considerar que o treinamento também é caracterizado como uma ferramenta de GC.

\subsubsection{Mecanismos tecnológicos}

A relevância da tecnologia para a gestão do conhecimento pode ser suportada pela grande quantidade de conhecimento disponível, a distribuição geográfica dos recursos e também dos consumidores, e ambientes dinâmicos (HASHEMI; KHADIVAR; SHAMIZANJANI, 2018). O Quadro 2 apresenta um resumo dos mecanismos tecnológicos associados aos autores que mencionam os mesmos.

\begin{tabular}{|c|c|}
\hline $\begin{array}{l}\text { Mecanismos } \\
\text { Tecnológicos }\end{array}$ & Autores \\
\hline Blogs & Sarka; Ipsen, 2017; O’Sullivan, 2007 \\
\hline Videoconferências & $\begin{array}{l}\text { Gomes; Oliveira; Chaves, 2018; Sarka; Ipsen, 2017; Kankanhalli et } \\
\text { al., 2003; Saito; Umemoto; Ikeda, 2007; Hansen; Nohria; } \\
\text { Tierney,1999; Bollinger e Smith, 2001; Binney, 2001. }\end{array}$ \\
\hline $\begin{array}{l}\text { Fóruns de Discussões } \\
\text { Eletrônicos }\end{array}$ & $\begin{array}{l}\text { Hashemi; Khadivar; Shamizanjani, 2018; Kankanhalli et al., 2003; } \\
\text { Saito; Umemoto; Ikeda, 2007; O’Sullivan, 2007; Hansen; Nohria; } \\
\text { Tierney,1999; Bollinger e Smith, 2001; Binney, } 2001 .\end{array}$ \\
\hline $\begin{array}{l}\text { Mensagens } \\
\text { Instantâneas }\end{array}$ & $\begin{array}{l}\text { Gomes; Oliveira; Chaves, 2018; Chen } \text { et al., 2018; Sarka; Ipsen, } \\
\text { 2017; Hansen; Nohria; Tierney,1999; Jasimuddin, 2007; Saito; } \\
\text { Umemoto; Ikeda, } 2007\end{array}$ \\
\hline Telefones & $\begin{array}{l}\text { Sarka; Ipsen, 2017; Hansen; Nohria; Tierney,1999; Kankanhalli et } \\
\text { al., 2003; Jasimuddin, } 2007 .\end{array}$ \\
\hline E-mails & $\begin{array}{l}\text { Gomes; Oliveira; Chaves, 2018; Sarka; Ipsen, 2017; Saito; } \\
\text { Umemoto; Ikeda, 2007; Bollinger e Smith, 2001; Binney, 2001; } \\
\text { Jasimuddin, 2007; Kankanhalli et al., 2003. }\end{array}$ \\
\hline Sistemas Especialistas & Binney, 2001; Benbya e Belbaly, 2005. \\
\hline Intranet & $\begin{array}{l}\text { Sarka; Ipsen, 2017; Skok e Kalmanovitch, 2005; Benbya e Belbaly, } \\
\text { 2005; Binney, 2001; Bollinger e Smith, 2001; Jasimuddin, 2007; } \\
\text { O’Sullivan, 2007; Saito; Umemoto; Ikeda, 2007. }\end{array}$ \\
\hline Repositórios & $\begin{array}{l}\text { Kankanhalli et al., 2003; Benbya e Belbaly, 2005; Binney, 2001; } \\
\text { Hansen; Nohria; Tierney, } 1999 .\end{array}$ \\
\hline Wiki & $\begin{array}{l}\text { Gomes; Oliveira; Chaves, 2018; Sarka; Ipsen, 2017; O’Sullivan, } \\
\text { 2007; Grace, 2009; Bollinger e Smith, 2001; Saito; Umemoto; } \\
\text { Ikeda, 2007. }\end{array}$ \\
\hline Páginas Amarelas & $\begin{array}{l}\text { Hashemi; Khadivar; Shamizanjani, 2018; Kankanhalli et al., 2003; } \\
\text { Bollinger e Smith, 2001; Hansen; Nohria; Tierney, } 1999 .\end{array}$ \\
\hline
\end{tabular}

Quadro 2 - Mecanismos tecnológicos para o compartilhamento do conhecimento.

Fonte: Adaptado de Oliveira, Maçada e Curado (2014).

Um dos mecanismos tecnológicos básicos citados por O'Sullivan (2007) é o diário eletrônico publicado na web, conhecido como blogue, que oferece uma forma barata de fornecer informações, fácil de criar, e podendo ser utilizada como uma fonte de informação juntamente com outras ferramentas para melhorar a comunicação. Kankanhalli et al. (2003) afirmam que são importantes as combinações de diretórios como ferramentas colaborativas, 
pois ajudam os funcionários a juntarem os seus conhecimentos e se conectarem. Os autores citam como exemplos de diretórios colaborativos as videoconferências e os fóruns de discussões eletrônicos, que permitem a rápida postagem de tópicos de discussão, consultas e soluções. Mecanismos tecnológicos podem suportar mecanismos não tecnológicos, como é o caso dos diretórios colaborativos que podem ser utilizados para suportar a comunidade de prática.

Jasimuddin (2007) afirma que as trocas de mensagens instantâneas permitem que os funcionários compartilhem experiências dentro de uma organização. Saito, Umemoto e Ikeda (2007) definem a comunicação como a funcionalidade principal da troca de e-mails, videoconferências, fóruns de discussões e mensagens instantâneas. Para uma ajuda mais direta na solução de problema, Binney (2001) explica que existem sistemas especialistas que possuem o objetivo de solucionar problemas com base em casos semelhantes registrados, e que esses sistemas são proativos ao retornar ao usuário com perguntas para restringir a busca de casos semelhantes.

A intranet também se destaca como uma ferramenta de grande relevância para o CC, visto que possui o objetivo de adquirir, armazenar e disseminar as informações para um grupo definido, além de ajudar a manter as conexões corretas entre os diferentes grupos, comunicando regras e procedimentos organizacionais (SKOK; KALMANOVITCH, 2005). Kankanhalli et al. (2003) apresentam os repositórios como outro mecanismo que facilita o CC dentro da organização. A Wiki se destaca por permitir que os funcionários de uma organização compartilhem o conhecimento em formato colaborativo e coletivo, permitindo ligar, publicar e editar documentos coletivamente (O'SULLIVAN, 2007).

Além dos softwares que colaboram para o CC, também é relevante que as organizações possuam maneiras de facilitar a localização dos funcionários especialistas em determinados assuntos. Em relação a isso, Kankanhalli et al. (2003) informam a existência de páginas amarelas, que são páginas onde há o mapeamento de tópicos organizacionais e os seus respectivos especialistas. Kankanhalli et al. (2003) relatam a importância destas páginas para localizar os colegas que possuem conhecimentos em problemas específicos, além de possibilitar formas de comunicação mais pessoais para obter o conhecimento dos especialistas.

\subsection{SELEÇÃO DE MECANISMOS}

Considerando que a competividade é alcançada utilizando o conhecimento organizacional, torna-se relevante o investimento por parte das organizações com relação à GC e com a valorização dos indivíduos que compõem a organização (JORGE; FALÉCO, 2016). Assim, é importante observar de que forma a empresa avalia os custos relacionados à GC e aos mecanismos de CC, pois a falta de investimento nessa área também acarreta custos que podem ser associados à manutenção do colaborador, como a falta de engajamento, falta de integração, entre outros. Portanto, para selecionar os mecanismos de CC é preciso averiguar se a GC da empresa está voltada para a estratégia organizacional, a fim de verificar se os mecanismos já existentes estão direcionando esforços corretamente.

$\mathrm{O}$ conjunto de mecanismos de $\mathrm{CC}$ selecionado deve atender tanto à estratégia de codificação quanto à de personalização, mesmo que em intensidades diferentes (HANSEN; NHORIA; TIERNEY, 1999). O custo de implantar uma nova tecnologia pode ser alto, portanto, utilizar das ferramentas já existentes para implantar os mecanismos de CC é relevante. Além das tecnologias utilizadas, é necessário levantar quais mecanismos não tecnológicos de compartilhamento de conhecimento já estão sendo utilizados pela organização.

Saito, Umemoto e Ikeda (2007) apresentam componentes que devem ser levados em consideração na escolha de mecanismos de CC, e agrupam cada um deles de acordo com a 
estratégia de GC que suporta. O Quadro 3 mostra os componentes e as estratégias apresentados pelos autores.

\begin{tabular}{|l|l|}
\hline \multicolumn{1}{|c|}{ Componentes } & \multicolumn{1}{c|}{ Estratégias que suportam } \\
\hline $\begin{array}{l}\text { Comunicação, distribuição, aprendizagem por } \\
\text { mídia eletrônica, colaboração e comunidade }\end{array}$ & Personalização \\
\hline Conectividade e Autoria & Personalização e Codificação \\
\hline $\begin{array}{l}\text { Armazenamento, pesquisa, fluxo de trabalho, } \\
\text { organização e raciocínio }\end{array}$ & Codificação \\
\hline
\end{tabular}

Quadro 3 - Componentes e estratégias suportadas. Fonte: Elaborado pelas autoras.

A comunicação é fundamental para transferir conhecimento entre as pessoas através dos processos de interação dentro de uma organização, da mesma forma que a relação entre o conhecimento e a comunicação é de grande importância para que as organizações possam se desenvolver continuamente (MACIEL; PIMENTEL; MARCHORI, 2013). A comunicação pode ser informacional ou relacional, mas o sucesso de ambas depende da intensidade das redes formais e informais, que são responsáveis por facilitar a disseminação de informações e os processos de interação entre os indivíduos da organização (MACIEL; PIMENTEL; MARCHORI, 2013).

Outra dimensão que foi analisada, de acordo com os componentes agrupados por Saito, Umemoto e Ikeda (2007), foi a distribuição do conhecimento.

$\mathrm{O}$ armazenamento do conhecimento é identificado como um processo da GC, e utilizando tecnologias da informação se pode reduzir o tempo de busca por determinado conteúdo (JORGE; FALÉCO, 2016). Jasimuddin (2005) afirma que é inadequado transferir conhecimento sem armazená-lo para utilização futura, da mesma forma que as organizações que armazenam seu conhecimento e posteriormente não o utilizam estão desperdiçando recursos e oportunidades de se obter vantagem competitiva através do conteúdo armazenado.

Visto que a organização pode ganhar vantagem competitiva através do CC entre os indivíduos, assim como com o devido armazenamento do conhecimento para utilização posterior (JASIMUDDIN, 2005), o que só é possível obter através da colaboração dos indivíduos que compõem a organização. A colaboração é identificada como um dos componentes abordados por Saito, Umemoto e Ikeda (2007) e é relevante, pois somente com a colaboração dos membros da organização será possível compartilhar e armazenar o conhecimento de forma adequada a fim de se obter vantagem.

A conectividade citada por Saito, Umemoto e Ikeda (2007), pode ser identificada como sendo a participação da organização em redes que facilitam o acesso, armazenamento, compartilhamento e integração do conhecimento (DUARTE et al. 2006). Pode-se identificar a internet como sendo uma das funcionalidades da conectividade (SAITO; UMEMOTO; IKEDA, 2007), caracterizando-se por ser o meio pelo qual a organização pode ligar pessoas, unidades, além de fazer o conhecimento fluir (DUARTE et al. 2006).

Através das comunidades constituídas de indivíduos com interesses em comum é possível construir e rever conhecimentos coletivamente através de um processo interativo (SILVA et al., 2012). Jasimuddin (2005) afirma que uma organização é um sistema constituído de conhecimento, e que ele está inserido em contextos organizacionais e comunidades particulares. O autor ressalta a importância do CC individual para outros membros a fim de beneficiar a organização, assim como, afirma que é necessário armazenar o conhecimento para torná-lo mais valioso com a possibilidade de utilizá-lo no presente e 
futuro. Saito, Umemoto e Ikeda (2007) também citam a aprendizagem por mídia eletrônica, e que pode ser utilizado no ambiente organizacional.

Além disso, o componente denominado como raciocínio pode ser caracterizado como sendo os sistemas que procuram solucionar casos com base em registros (BINNEY; 2001). Esses sistemas se baseiam em regras, casos, bases de conhecimento etc. (SAITO; UMEMOTO; IKEDA, 2007). Além dos sistemas de raciocínio que podem ser utilizados pela organização, Saito, Umemoto e Ikeda (2007) também citam os sistemas de busca, que denominam como o componente chamado de pesquisa. Através dos sistemas de pesquisa é possível localizar indivíduos da organização com o conhecimento necessário para resoluções de problemas, e aproximar a comunicação com o objetivo de se absorver maior conhecimento dos especialistas (KANKANHALLI et al., 2003).

Por fim, a organização deve garantir a disponibilização do conhecimento para qualquer membro que o necessite, em qualquer lugar, usando ou não tecnologias (BOSE, 2004), com o objetivo de se obter maior aproveitamento do conhecimento e ganhar competitividade. Portanto, a organização também se caracteriza um componente (SAITO; UMEMOTO; IKEDA, 2007) que deverá ser analisado para escolha de mecanismos de CC.

\section{MÉTODO}

Esta seção apresenta a classificação da pesquisa (seção 3.1), coleta de dados (seção 3.2) e análise de dados (seção 3.3).

\subsection{CLASSIFICAÇÃO DA PESQUISA}

Esta pesquisa, quanto à abordagem, caracteriza-se como qualitativa, pois há preocupação de se entender o caso, conforme indicado por Flick (2009), sua natureza é aplicada e tem objetivo exploratório. Esta pesquisa se caracteriza como estudo de caso único nas áreas administrativas corporativas de uma indústria brasileira do ramo de pneus e borrachas. A competitividade existente na indústria de artefatos de borrachas e pneumáticos, a sua participação no PIB, e a forma como os seus resultados afetam outros setores, são características relevantes para aplicação da pesquisa em uma organização deste ramo. $\mathrm{O}$ fato de se ter no Brasil uma das principais fabricantes mundiais de produtos para reforma e reparos de pneus e câmeras de ar, e a sua abrangência nacional e atuação internacional foram critérios considerados para a seleção do caso, sabendo-se que o CC neste setor é de suma importância para potencializar as ações no mercado. Aliado a isto, na escolha do caso também foi considerada a facilidade de acesso às informações por uma das autoras ser funcionária da empresa.

\subsection{COLETA DE DADOS}

Os dados foram coletados através de observação direta e entrevistas semiestruturadas. As entrevistas foram realizadas com funcionários de diferentes cargos e níveis hierárquicos, até se obter a saturação por nível hierárquico agregado (operacional, tático e estratégico), ou seja, quando o último entrevistado não trouxe contribuições novas em relação às perguntas realizadas. A coleta de dados ocorreu no período de agosto a setembro do ano de 2017. A empresa possui 1900 funcionários distribuídos em diferentes estados do Brasil. No entanto, esta pesquisa foi realizada na sede da empresa que fica no Rio Grande do Sul, e conta com 160 funcionários. O objetivo de entrevistar diferentes cargos foi avaliar a percepção de cada nível hierárquico agregado sobre os mecanismos de CC presentes na organização, a fim de se verificar o nível de satisfação dos mesmos, e selecionar outros mecanismos que possam suprir as necessidades existentes e alavancar a estratégia da empresa. Não foi permitido detalhar o perfil dos entrevistados, sendo identificados na análise de dados apenas pelo nível hierárquico 
agregado. O nível estratégico está composto por gerentes e diretores (2 níveis hierárquicos), o nível tático por coordenadores e especialistas (2 níveis hierárquicos), e o operacional por assistentes, analistas e compradores (3 níveis hierárquicos). A relação dos entrevistados e nível hierárquico é apresentada no quadro a seguir.

\begin{tabular}{|c|c|}
\hline Identificação dos Entrevistados & Nível Hierárquico Agregado \\
\hline $1,2,4,8,10$ até 17 e 19 & Operacional \\
\hline 3,7 e 9 & Tático \\
\hline 5,6 e 18 & Estratégico \\
\hline
\end{tabular}

Quadro 4 - Relação de entrevistados com identificação do nível hierárquico.

Fonte: Elaborado pelas autoras.

O roteiro da entrevista semiestruturada foi elaborado com base nos critérios apresentados na revisão de literatura. As questões consideradas abrangeram: os componentes relevantes; os mecanismos existentes; os pontos positivos e negativos dos mecanismos existentes; a necessidade de novos mecanismos; a relação dos mecanismos com as estratégias de codificação e personalização; investimento da empresa na gestão e compartilhamento do conhecimento. O roteiro de entrevista foi analisado por dois pesquisadores de GC, que sugeriram ajustes quanto ao enunciado de algumas questões. As entrevistas foram realizadas presencialmente, face a face, no próprio ambiente da empresa, com duração média de uma hora. As entrevistas foram gravadas com a permissão dos entrevistados e posteriormente transcritas para auxiliar na análise dos dados. A observação foi participante e não estruturada, realizada por uma das autoras que trabalha na empresa. A observação foi realizada no período de abril a setembro do ano de 2017. Neste período foram realizadas anotações sobre o tópico e aspectos relacionados a ele, sempre que o mesmo aparecia em conversas informais e reuniões.

\subsection{ANÁLISE DE DADOS}

A técnica escolhida para realizar a análise das entrevistas foi a análise de conteúdo. $\mathrm{O}$ conteúdo das observações foi confrontado com a análise das entrevistas com o intuito de verificar ou explicar o resultado obtido através das entrevistas, e desta forma permitindo a triangulação dos dados.

A análise de conteúdo se caracteriza como um conjunto de técnicas para analisar as comunicações utilizando procedimentos sistemáticos para descrever o conteúdo obtido das mensagens, considerando que o interesse não está na descrição, mas sim, no aprendizado obtido através do conteúdo tratado (BARDIN, 2009). A autora ressalta que a enumeração das características de forma resumida e o seu tratamento é a primeira etapa necessária da análise do conteúdo, e o significado concedido a essas características é a última, sendo que a inferência é o procedimento que faz a passagem explícita entre ambas as etapas.

A análise do conteúdo é composta pela pré-análise, exploração do material, tratamento dos resultados, a inferência e a interpretação (BARDIN, 2009). A pré-análise é a fase de organização, onde a transcrição das entrevistas foi organizada, assim como as anotações da observação. Após esta etapa, foi realizada a exploração do material, que consistiu na leitura e codificação dos dados. A codificação foi realizada considerando as perguntas, sendo que os códigos iniciais foram relacionados a partir da revisão da literatura, e o critério adotado foi o de presença ou ausência em cada entrevista. O código foi considerado uma vez, mesmo que o entrevistado tenha mencionado o mesmo mais de uma vez durante a entrevista. Nas entrevistas não foram identificados novos códigos. Por último, foi realizada a interpretação, considerando as observações, que permitiu a elaboração de quadros de resultados e as inferências. 
Os dados obtidos através das entrevistas e da observação direta foram analisados de forma a avaliar quais mecanismos de compartilhamento de conhecimento a organização já adota, se são satisfatórios na percepção dos usuários, e quais poderão ser implantados seguindo a estratégia da organização para suprir as necessidades existentes.

\section{ANÁLISE DE DADOS E DOS RESULTADOS}

Esta seção está dividida em subseções que demonstram os resultados obtidos nas entrevistas e observações.

\subsection{COMPONENTES IMPORTANTES PARA SELEÇÃO DE MECANISMOS}

A análise das entrevistas realizadas demonstrou que a comunicação é de extrema relevância para praticamente todos os funcionários da organização, e teve destaque na maior parte das respostas obtidas. Eles a consideram essencial para que a transferência de conhecimento ocorra e seja bem-sucedida, o que está alinhado com o apresentado por Maciel, Pimentel e Marchori (2013). "O domínio da comunicação é imprescindível para transmitir o conhecimento. É importante para fazer a pessoa que está aprendendo absorver o que está sendo transmitido" (ENTREVISTADO 09).

É correto afirmar que a comunicação é de grande relevância para os entrevistados, porém, através das observações foi identificado que o motivo pelo qual este componente foi enfatizado tantas vezes durante as entrevistas está relacionado com o fato de a empresa ter investido recentemente na contratação de um profissional de comunicação interna e endomarketing. Conforme citado em algumas entrevistas, o novo profissional reformulou a comunicação interna existente e vem implantando novos trabalhos que geram grandes impactos e são bem vistos pelos colaboradores.

A análise demonstrou que a colaboração foi identificada pelos níveis operacionais e táticos como sendo o mais importante após a comunicação. Alguns dos entrevistados acreditam que a colaboração deveria ser trabalhada melhor entre os colegas, pois evitaria que algumas pessoas ficassem sobrecarregadas de trabalho em períodos que outras estão ociosas. No entanto, a maior parte que citou este componente como importante alegou que a comunicação e a colaboração estão relacionadas, pois a comunicação pode alinhar as informações e fazer com que as pessoas trabalhem por um mesmo objetivo através da colaboração umas com as outras, como se observa na fala do Entrevistado 03, de nível tático "[...] isso é uma coisa que nós estamos trabalhando cada vez mais, essa coisa de um colaborar com o outro para chegar num resultado".

Bose (2004) menciona que a organização é importante por garantir a disponibilização do conhecimento. Quanto a este componente, os entrevistados de nível operacional e tático disseram que é importante por promover e estimular os colaboradores quanto assuntos referentes à $\mathrm{GC}$, facilitar o $\mathrm{CC}$, e apoiar os colaboradores na busca por desafios. "A empresa tem um papel importantíssimo na GC, porque às vezes falta estímulo nas pessoas e a empresa pode auxiliar para que isso aconteça com mais frequência, promovendo isso" (ENTREVISTADO 09). Porém, para outros entrevistados, a importância deste componente está relacionada à organização dos processos, fluxos de trabalho, e organização das informações necessárias para desempenhar as atividades. Este componente não foi considerado importante por nenhum entrevistado de nível estratégico.

Os componentes de comunidade, conectividade, armazenamento e fluxo de trabalho foram citados como importante por menos entrevistados em comparação aos componentes de comunicação e colaboração. A visão dos entrevistados que mencionaram a comunidade está de acordo com o que foi citado por Silva et al. (2012). "Comunidade é importante porque tu pegas representantes de determinadas áreas, e reuni o conhecimento de 
todos que estão ligados àquele projeto" (ENTREVISTADO 06). Já a conectividade foi mencionada nas entrevistas conforme apresentado por Duarte et al. (2006).

Os entrevistados que consideraram importante o armazenamento, afirmaram que este componente é importante para reter o conhecimento na organização, e o que foi mencionado por eles está de acordo com Jasimuddin (2005). "Acho que o armazenamento também é muito importante porque tem muita coisa que fica na cabeça das pessoas, e as pessoas vão embora e o conhecimento vai embora" (ENTREVISTADO 13). Já o componente fluxo de trabalho, foi considerado importante pelos entrevistados por se tratar de uma forma de organizar as atividades e os processos, e desta forma enxergar as melhorias. Eles também citam que o fluxo de trabalho retém conhecimento sobre as atividades desenvolvidas, minimizando o impacto gerado pela rotatividade que ocasiona a perda de informações e de processos que não estão documentados. "Fluxo de trabalho ajuda na rotatividade, quando uma pessoa não estiver mais na empresa o que ela fazia está descrito para o processo não se perder" (ENTREVISTADO 12).

Foi identificado na análise de dados, que o nível estratégico em sua totalidade, considera a comunicação como o componente mais importante, assim como os demais níveis. Porém, nenhum dos entrevistados do nível estratégico considerou a organização, que seria o terceiro componente mais importante para os níveis operacionais e táticos, um componente importante. Para este nível hierárquico, os componentes mais importantes após a comunicação seria o armazenamento, fluxo de trabalho e raciocínio. Este fato, de acordo com a observação, está relacionado à diretriz da empresa no momento das entrevistas, que estava focada em rever e mapear processos, fluxos de trabalho e na codificação, para atender o novo item da norma ISO 9001:2015 sobre conhecimento organizacional.

Os demais componentes como distribuição, pesquisa, raciocínio, e aprendizado por mídia eletrônica foram citados por poucos entrevistados, enquanto a autoria não foi considerada importante por nenhum deles.

\subsection{MECANISMOS DE CC IDENTIFICADOS}

Os mecanismos de CC apontados pelos entrevistados para atender os componentes considerados mais importantes, são os mesmos que eles utilizam no dia a dia, e que foram identificados que a empresa possui. Alguns dos mecanismos citados foram treinamentos, repositório que faz o gerenciamento de documentos, telefone, mensagens instantâneas (WhatsApp e Lync), videoconferência e diretórios.

A intranet, apesar de estar em fase de implantação na empresa pesquisada, é considerada pelos entrevistados um importante mecanismo para centralizar algumas informações e processos, e isso está de acordo com o que foi abordado por Skok e Kalmanovitch (2005). O Entrevistado 12 afirma “[...] vai fazer muita diferença para nós, é um canal onde tu vai centralizar todas as informações. Hoje trabalhamos com canais pequenos que não possuem todas as informações e não atingem a todos". A intranet também foi o mecanismo citado pela maioria dos entrevistados quanto aos mecanismos que faltam na organização. "O que estava faltando virá com a intranet, pois irá centralizar a informação de massa para que fique mais rapidamente acessível e que permita a interação" (ENTREVISTADO 09). A observação revela que o lançamento deste mecanismo foi informado aos colaboradores recentemente, e que a expectativa criada pela divulgação da implantação que está sendo realizada, faz com que os entrevistados se lembrem deste mecanismo quando questionados sobre o que faltava na organização. Além, da experiência de alguns dos entrevistados com essa ferramenta em outras empresas. Porém, a intranet não foi identificada nas respostas de um número significativo de entrevistados quando questionados sobre os mecanismos existentes na organização, pois eles ainda não haviam interagido com o mecanismo que estava sendo implantado. Consequentemente, a maior parte deles não 
associou o mecanismo ao atendimento aos componentes considerados importantes, visto que citaram os mecanismos utilizados no dia a dia.

Apesar de o e-mail ter sido citado pelo maior número de entrevistados, tanto quanto ao que existe na organização, quanto ao que atende aos componentes considerados importantes, ele não foi o mecanismo que obteve maior destaque nas respostas a estes questionamentos. A comunicação face a face, como as reuniões apresentados por Hansen, Nohria e Tierney (1999), que se caracteriza conversão de conhecimento através da socialização, conforme apresentado por Nonaka e Takeuchi (2008), foram as mais citadas como sendo o mecanismo mais eficiente quando se trata de compartilhar informações e conhecimento. Conforme citou o Entrevistado 10 "Eu acho que falando pessoalmente às vezes tu consegue expressar melhor o que tu quer dizer do que escrito ou de qualquer outra forma. A percepção que a pessoa tem face a face é muito melhor". O e-mail foi mencionado nas respostas obtidas, como apresentado pelo Entrevistado 07 "[...] para mim funciona assim, ou face a face, ou eu ligo e depois formalizo por e-mail". Essa formalização do que foi acordado por e-mail foi muito citada durante as entrevistas, e pode ser identificada como conversão do conhecimento através da externalização, apresentada por Nonaka e Takeuchi (2008).

O gerenciador de documentos obteve destaque nas respostas do nível estratégico quanto aos questionamentos sobre os mecanismos que existem na organização, e sobre os mecanismos aptos a atender aos componentes considerados importantes. $\mathrm{O}$ pesquisador observou que este fato também está relacionado à diretriz da organização, citada na seção 4.1, pois os fluxos de trabalhos, mapeamento de processos, dentre outros documentos, são armazenados no gerenciador de documentos, que foi implantado recentemente pela organização.

Ao final das entrevistas foram apresentados todos os mecanismos tecnológicos e não tecnológicos levantados no referencial teórico, e questionado sobre quais mecanismos os entrevistados acreditavam que a empresa não possui atualmente, e que seriam úteis. A maior parte dos entrevistados não identificou a utilização de blogue e de fórum de discussão eletrônico na organização, e apenas alguns informaram que esses mecanismos poderiam ser úteis, assim como a Wiki. Porém, a Wiki gerou dúvida sobre se as pessoas estão preparadas para editar documentos de forma colaborativa, e sobre quem seria habilitado a editar os documentos. Em contrapartida, a Wiki facilitaria o trabalho de pessoas que atualmente trabalham em conjunto e não possuem mecanismo específico para isso, o que está de acordo com O’Sullivan (2007) e Grace (2009). Já o mentoring foi identificado por alguns entrevistados como sendo um mecanismo muito importante, mas existente somente nas áreas preocupadas com o que foi apresentado por Lawrence (2008), e por iniciativa própria, sem o incentivo da empresa, apesar de beneficiá-la.

Muitos dos entrevistados acreditam que a área de tecnologia da informação possui um sistema especialista como o que foi apresentado por Binney (2001), e que este sistema seria importante para outras áreas manterem um histórico sobre a resolução de problemas. As páginas amarelas foram as mais apontadas como benéficas para a organização, pois a maior parte dos entrevistados relata a dificuldade de localizar os especialistas de determinados assuntos. "Páginas amarelas seriam maravilhosas porque a gente não perderia tanto tempo tentando adivinhar quem é o responsável, ali já estaria especificado os contatos" (ENTREVISTADO 01), exatamente como apontado por Kankanhalli et al. (2003).

\subsection{PONTOS POSITIVOS E NEGATIVOS DOS MECANISMOS IDENTIFICADOS NA ORGANIZAÇÃO}

Alguns mecanismos tiveram pontos negativos e positivos citados de forma significativa, e serão abordados nesta subseção. O Lync foi bastante mencionado entre os entrevistados, que alegam que este mecanismo facilita a comunicação, é rápido e ágil, e 
possibilita o registro, o que está de acordo com Jasimuddin (2007) e Saito, Umemoto e Ikeda (2007). Porém, o ponto negativo mais citado está relacionado ao fato de que nem todos os funcionários possuem esta ferramenta, além de não poder ser utilizada com fornecedores, e poder ser utilizada inadequadamente pelos funcionários. "O Lync é rápido, mas em compensação não são todas as pessoas que tem, assim como, infelizmente deve ter pessoas que utilizam para fins que não são trabalho" (ENTREVISTADO 17).

$\mathrm{O}$ e-mail permite registrar e armazenar o que foi enviado, fornece garantia sobre as informações fornecidas, além de poder comunicar várias pessoas ao mesmo tempo. Mas, o tamanho de armazenamento das caixas de e-mails, a tecnologia da organização que permite quedas de e-mails por grandes períodos de tempo, a má interpretação ou entendimento dos destinatários dos $e$-mails, e o fato das pessoas não conseguirem visualizar as expressões e interagirem sobre assuntos, foram apresentados como pontos negativos deste mecanismo. " $\mathrm{O}$ e-mail não tem sentimentos, expressão, não tem como tirar dúvidas. Quando se passa uma legislação é sabido que talvez se tenha que ligar para explicar" (ENTREVISTADO 03).

A videoconferência permite conectar pessoas sem precisar marcar viagens, e possibilita ver expressões, facilitando a comunicação, conforme afirmado por Saito, Umemoto e Ikeda (2007). Porém, causa desconforto para as pessoas que ficam se observando pela televisão, não permite ver o problema no seu local e necessita de tempo na agenda dos envolvidos. "Se estivermos discutindo um assunto de uma máquina, na videoconferência tu não poderá ver a máquina" (ENTREVISTADO 05). O telefone também não possibilita enxergar o problema, o que pode dificultar a explicação, assim como, não permite ver expressões, e não possibilita o registro, mas é esclarecedor e útil para resolver assuntos rápidos.

As reuniões (HANSEN; NOHRIA; TIERNEY, 1999) foram as mais apontadas como positivas, pois permitem ver expressões, facilita a resolução de problemas, propicia o debate e o questionamento. No entanto, também precisa da agenda dos participantes, e muitas vezes falta objetividade e preparação dos envolvidos.

O gerenciador de documentos foi elogiado por armazenar os processos das áreas, por permitir que as pessoas saibam onde estão armazenados os documentos e se estão atualizados, assim como, facilitam os treinamentos das áreas, que podem realizar leituras individuais. Isto está de acordo com o que foi afirmado por Kankanhalli et al. (2003) sobre o reuso do conhecimento. O Entrevistado 13 ressalta que "[...] ao invés de colocarmos um monte de gente numa sala para ficar falando de forma maçante, todo mundo vai ler quando puder, e fica registrado". Para este mecanismo não foram identificados pontos negativos por muitos entrevistados, somente que não houve uma apresentação adequada da ferramenta, e sobre a dificuldade de encontrar os documentos pela forma como as pastas deste repositório foram organizadas.

\subsection{FINALIDADES DOS MECANISMOS IDENTIFICADOS}

Atingir e melhorar os resultados, apenas compartilhar informações, ou compartilhar as informações com o objetivo de desempenhar e facilitar os processos, assim como, melhorar a comunicação e a integração, foram as finalidades mais identificadas pelos entrevistados para os mecanismos presentes na organização. Como, por exemplo, pode-se identificar na fala do entrevistado: "A principal finalidade é facilitar o andamento dos processos, fazendo com que as pessoas consigam atingir os objetivos que a empresa propõe" (ENTREVISTADO 06).

Outras finalidades como a redução de custos, transparência nos processos, maior agilidade e troca de informações para auxiliar a tomada de decisões também foram citadas por uma parte menor dos entrevistados, como apontado pelo Entrevistado 12 “[...] ajudam a tomar decisões com colegas e fornecedores que estão distantes". 
A grande maioria dos entrevistados afirmou que os mecanismos presentes atendem às finalidades propostas pela organização. Como o Entrevistado 02, que apontou uma melhora significativa na comunicação em nível empresa, e afirma que "[...] se tu não comunicas, a pessoa fica sabendo do mesmo jeito. E às vezes, uma notícia que deveria ter chegado de uma forma, chega de outra". Entretanto, uma parte dos entrevistados, alegou que os mecanismos atendem parcialmente, como o Entrevistado 13, que identifica mecanismos tecnológicos que poderiam ajudar a organização a reduzir custos com deslocamentos de funcionários e fornecedores, mas que não são liberados pela questão da segurança da informação. Já o Entrevistado 17 acredita que os mecanismos presentes viabilizam as finalidades propostas, mas que não atingem por completo. Ele afirma que a empresa deveria estimular a comunicação entre as pessoas para "que elas não tenham medo de falar, que elas falem o que pensam com respeito, [...] a empresa pode se surpreender com as pessoas que ficam quietinhas e que podem colaborar bastante" (ENTREVISTADO 17).

Quando se trata do investimento por parte da empresa em gestão e CC, o nível estratégico, a maior parte do nível tático e parte do nível operacional afirmam que existe, ou que a organização começou a se preocupar com isso há pouco tempo. Enquanto que, a maior parte do nível operacional afirma que não existe uma preocupação direta da empresa, mas sim de pessoas de determinadas áreas que se preocupam com isso pelas experiências que tiveram. Eles alegam que os investimentos feitos pela empresa são para a melhora dos processos, e que a disseminação das informações e do conhecimento é uma consequência disso. "A empresa investiu nesses mecanismos para cumprimento de certificações, [...], mas não pensando em gestão do conhecimento e para alcançar os resultados que se teria com uma gestão" (ENTREVISTADO 01). O Quadro 5 apresenta a relação entre número de entrevistados e o investimento da empresa com relação ao CC.

\begin{tabular}{|l|c|c|c|c|}
\hline Nível Hierárquico & Operacional & Tático & Estratégico & Total \\
\hline Total entrevistados & $\mathbf{1 3}$ & $\mathbf{3}$ & $\mathbf{3}$ & $\mathbf{1 9}$ \\
\hline $\begin{array}{l}\text { Não incentiva o compartilhamento, possui dificuldade } \\
\text { de reter conhecimento, ou não investe em gestão do } \\
\text { conhecimento. }\end{array}$ & 7 & 1 & $\mathbf{0}$ & 8 \\
\hline $\begin{array}{l}\text { Começou a se preocupar com isso há pouco tempo, ou } \\
\text { investe e se preocupa com o CC. }\end{array}$ & 6 & 2 & $\mathbf{3}$ & 11 \\
\hline
\end{tabular}

Quadro 5 - Investimento da empresa com relação ao CC.

Fonte: Elaborado pelas autoras.

\subsection{ESTRATÉGIAS DE CODIFICAÇÃO E PERSONALIZAÇÃO}

As respostas obtidas revelam que existem ambas as estratégias apresentadas por Hansen, Nohria e Tierney (1999) na organização, mas que atualmente prevalece a estratégia de codificação sobre a de personalização. Os entrevistados com mais tempo de empresa informaram que no passado a empresa trabalhava intensamente com a estratégia de personalização, e essa estratégia dominante acarretou a perda de informações e conhecimento com a rotatividade. Diante desse contexto, os entrevistados notam a preocupação e incentivo por parte da empresa em codificar todos os processos e informações possíveis, principalmente com a ajuda do gerenciador de documentos citado anteriormente. "Justamente por passar por um processo de perda de informações em função de ser somente personalizada que a empresa começou a codificar, para reter esse conhecimento que antes era difundido só entre as pessoas" (ENTREVISTADO 09). O Quadro 6 apresenta a relação entre o número de entrevistados e as estratégias aplicadas pela organização. 


\begin{tabular}{|l|c|c|c|c|}
\hline \multicolumn{1}{|c|}{ Nível Hierárquico } & Operacional & Tático & Estratégico & Total \\
\hline \multicolumn{1}{|c|}{ Total entrevistados } & $\mathbf{1 3}$ & $\mathbf{3}$ & $\mathbf{3}$ & $\mathbf{1 9}$ \\
\hline Codificação, ou ambas, com mais codificação & 7 & 2 & 2 & 11 \\
\hline Personalização, ou ambas, com mais personalização & 4 & 1 & 0 & 5 \\
\hline Ambas as estratégias na mesma proporção & 2 & 0 & 1 & 3 \\
\hline
\end{tabular}

Quadro 6 - Relação entre o número de entrevistados e as estratégias aplicadas pela organização.

Fonte: Elaborado pelas autoras.

Quando questionados sobre em qual estratégia a empresa deveria investir mais, as respostas se dividiram em ambas as estratégias em proporções iguais, e em mais personalização. Os entrevistados que entendem que deve haver maior investimento na estratégia de personalização alegam que existe conhecimentos que não são possíveis de codificar, seja porque as informações mudam com muita frequência, ou porque a personalização é utilizada no aprendizado do dia a dia. Alguns deles acreditam que a personalização ajuda no desenvolvimento do know-how, como apresentado pelo Entrevistado 14 "[...] se eu não vier mais, ninguém vai fazer o trabalho que eu faço da mesma maneira, e com o mesmo domínio que eu tenho".

Já os entrevistados que compreendem que deve haver ambas as estratégias em proporções iguais, alegam que a codificação é necessária para que o conhecimento e as informações sejam retidos na organização mesmo na ausência de determinadas pessoas, enquanto que a personalização tem a mesma importância por se tratar das experiências e criatividade de cada indivíduo. "Tem muitas áreas que se falam, mas não documentam o que está acontecendo, e isso é um risco porque a informação pode se perder. [...] para a organização é um risco deixar as coisas registradas somente na cabeça das pessoas" (ENTREVISTADO 18). Outras afirmam que a codificação é importante para manter histórico de processos na organização, conforme apresentado pelo Entrevistado 02, “[...] às vezes eu tento buscar históricos e não encontro. Até mesmo as pessoas, se não tem bastante tempo de empresa não sabem de nada". O Quadro 7 representa a relação entre o número de entrevistados e a estratégia que a empresa deveria adotar.

\begin{tabular}{|l|c|c|c|c|}
\hline Nível Hierárquico & Operacional & Tático & Estratégico & Total \\
\hline Total entrevistados & $\mathbf{1 3}$ & $\mathbf{3}$ & $\mathbf{3}$ & $\mathbf{1 9}$ \\
\hline $\begin{array}{l}\text { Personalização, ou } \\
\text { ambas, com mais personalização }\end{array}$ & $\mathbf{8}$ & 0 & 0 & $\mathbf{8}$ \\
\hline Ambas as estratégias na mesma proporção & 3 & $\mathbf{2}$ & $\mathbf{2}$ & $\mathbf{7}$ \\
\hline $\begin{array}{l}\text { Codificação, ou } \\
\text { ambas, com mais codificação }\end{array}$ & 2 & 1 & 1 & 4 \\
\hline
\end{tabular}

Quadro 7 - Relação entre o número de entrevistados e a estratégia que a empresa deveria adotar. Fonte: Elaborado pelas autoras.

A ISO 9001:2015 possui um item sobre conhecimento organizacional, o qual determina que a empresa armazene e disponibilize o conhecimento necessário para a realização de seus processos. Isto pode estar influenciando a estratégia de codificação adotada pela empresa, uma vez que a codificação torna a comprovação do novo item da ISO 9001:2015 mais fácil que a personalização. Embora nenhum dos entrevistados tenha associado a estratégia de codificação com o atendimento do item da norma.

\section{DISCUSSÃO DOS RESULTADOS}

O trabalho analisou componentes, mecanismos e estratégias para o compartilhamento 
do conhecimento na organização. Percebe-se que os entrevistados conseguem identificar a utilização das estratégias apresentadas por Hansen, Nohria e Tierney (1999) pela organização. No entanto, é possível concluir que a organização não possui uma estratégia de GC clara, com base nas finalidades identificadas na subseção 4.4. Dessa forma, as estratégias levantam duas preocupações entre os entrevistados. Uma delas se refere ao armazenamento dos processos e informações, que eles afirmam que se deve codificar para que estas não se percam e prejudiquem a empresa e quem permanece trabalhando nela. A outra se refere ao desenvolvimento das competências e qualidades de um especialista, que os entrevistados alegam ocorrer através da personalização, não sendo possível codificar. Desta forma, se os colaboradores estão acostumados e preferem realizar a conversão do conhecimento através da socialização, a empresa terá dificuldade em fazer com que estes codifiquem corretamente e fielmente o conhecimento organizacional. Neste caso, é importante investir em conscientização para que os colaboradores mantenham os conteúdos codificados atualizados para que possam ser utilizados quando necessário, além de estimular o aproveitamento deles em seus projetos ou processos diários. A implantação de uma estratégia de gestão e compartilhamento do conhecimento estimularia a colaboração, que conforme visto na seção 4.1, foi identificado como sendo um componente citado por Saito, Umemoto e Ikeda (2007) que precisa ser trabalhado entre os colegas e equipes.

Embora a empresa possua variados mecanismos de CC, definir uma estratégia de GC é primordial para selecionar os mecanismos mais adequados, e para que eles sejam aproveitados da melhor maneira possível, gerando mais eficiência para os processos. Este trabalho identificou mecanismos que a empresa poderia adicionar ao conjunto já existente para atender outros componentes citados por Saito, Umemoto e Ikeda (2007) considerados importantes na subseção 4.1, e as necessidades apontadas na subseção 4.2. Para atender ao componente comunicação, que foi identificada como o mais importante para esta organização, as páginas amarelas seriam de grande importância para suprir a necessidade existente quanto à localização dos especialistas de determinados assuntos. Enquanto que, os sistemas especialistas seriam úteis para manter o histórico de casos e resoluções e problemas, atendendo aos componentes de conectividade e armazenamento.

Foi identificado que o mentoring é um mecanismo de $\mathrm{CC}$ de grande relevância, o que justifica ser praticado por alguns colaboradores através da iniciativa própria, sem o incentivo da organização. Deste modo, a avaliação dos resultados obtidos pela utilização deste mecanismo também deve ser levada em consideração pela organização. Além disso, a implantação deste mecanismo atenderia ao componente organização, citado por Saito, Umemoto e Ikeda (2007). Como foi visto por Bose (2004), a organização deve garantir a disponibilização do conhecimento, e o mentoring atenderia este componente por apoiar e incentivar os colaboradores.

Percebe-se também que o objetivo da organização ao investir em mecanismos não foi diretamente a gestão e o compartilhamento do conhecimento organizacional, nem o utilizar estrategicamente através do potencial das pessoas. A falta dessa estratégia pode fazer com que a empresa tenha dificuldades de reter grandes talentos, pois as pessoas podem ter dificuldade de enxergar um crescimento pessoal e profissional dentro da organização. Além disso, pode fazer com que a empresa perca oportunidades de aproveitar melhor o seu conhecimento interno, através de pessoas que se sentindo valorizadas pelo seu conhecimento, ou pelo acompanhamento do seu desenvolvimento, poderiam contribuir mais.

Mecanismos considerados importantes e que não estão disponíveis para utilização de todos os funcionários foram identificados. Isto se justifica porque estes mecanismos possuem um custo elevado, e também pela utilização inadequada pelos funcionários. No último caso, há necessidade de a organização implantar políticas de uso de mecanismos. A política de uso 
também poderá ser aplicada, juntamente com treinamentos, para disponibilizar informações e modo adequado de utilização dos diversos mecanismos existentes na organização.

Dos componentes apresentados por Saito, Umemoto e Ikeda (2007), é notável a valorização da comunicação pelos entrevistados, seja pela sua importância no CC, ou pelos bons resultados proporcionados pela nova gestão. De qualquer forma, o que os resultados obtidos mostram é que a comunicação da empresa para com os funcionários melhorou consideravelmente, mas a comunicação entre os colegas de área e entre as equipes necessita ainda de atenção para que haja maior troca e colaboração entre as pessoas. Isso pode ser observado nos momentos em que os entrevistados sugeriram que a empresa estimule as pessoas a opinarem sobre o que pensam, alegando que a empresa pode se surpreender com a colaboração advinda desta iniciativa, ou quando mencionaram sobre as dificuldades de obter informações de outras áreas para o andamento dos processos, e com a afirmação sobre a falta de colaboração entre os colegas em momentos de muito trabalho.

Outros componentes também são importantes para os entrevistados: colaboração, organização, comunidade, conectividade, armazenamento e fluxo de trabalho. É interessante notar que as diretrizes da empresa influenciam os níveis hierárquicos de forma diferente, fazendo com que colaboração e organização não fossem citadas pelo nível estratégico, que valoriza mais os componentes de armazenamento e fluxo de trabalho. A figura seguinte traz uma proposta de seleção de mecanismos para compartilhamento do conhecimento na organização de acordo com os componentes mais valorizados.

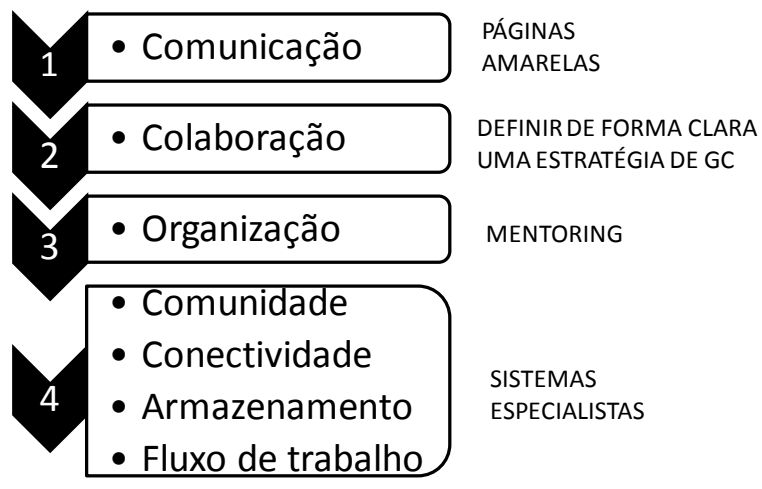

Figura 1 - Proposta da seleção de mecanismos

\section{CONSIDERAÇÕES FINAIS, LIMITAÇÕES E PESQUISAS FUTURAS}

O objetivo deste trabalho era selecionar mecanismos para o $\mathrm{CC}$ nas áreas administrativas corporativas de uma indústria de artefatos de borrachas e pneumáticos brasileira. Isto foi realizado através de uma pesquisa qualitativa exploratória, que utilizou entrevistas e observação participante como forma de coleta de dados. Os resultados mostram os componentes mais valorizados e sugerem os mecanismos de sistemas especialistas, páginas amarelas e incentivo ao mentoring, além da definição clara de uma estratégia de gestão do conhecimento.

Esta pesquisa contribui academicamente por se tratar de um estudo sobre mecanismos de $\mathrm{CC}$ realizado na área da indústria brasileira de pneus e borrachas, um contexto que ainda apresenta oportunidades para ser explorado. Colabora também com a literatura da gestão do conhecimento, ao mostrar o processo de escolha dos mecanismos. E colabora com a prática, pois gerentes de empresas, em especial do setor de borrachas, podem utilizar os resultados dessa pesquisa para implementar processos e mecanismos de gestão do conhecimento. 
Esta pesquisa ficou restrita, quanto à unidade de análise, nas áreas administrativas corporativas de uma indústria brasileira do ramo de pneus e borrachas. Essa restrição ocorreu porque as fábricas não possuem a mesma metodologia de trabalho que as áreas administrativas, nem a mesma tecnologia. As áreas administrativas localizadas em Porto Alegre são denominadas corporativas por comportarem a alta administração, e por possuírem a responsabilidade de alinhar a estratégia de negócio estabelecida por eles com as demais unidades do grupo de empresas. Desta forma, as áreas administrativas corporativas tiveram maior relevância para esta pesquisa, pois estão diretamente ligadas às diretrizes da alta gestão e contribuem para a análise dos mecanismos de CC existentes, assim como, para entender a necessidade da empresa e identificar outros mecanismos que contribuam para a estratégia da mesma.

Pesquisas futuras deverão considerar a utilização de métodos mistos para coleta de dados, como a aplicação de questionários após a realização de entrevistas para complementar as respostas obtidas. As entrevistas semiestruturadas permitem que os entrevistados discorram sobre os assuntos que serão abordados, os guiando para que não percam de vista os assuntos que deverão ser tratados. Porém, a liberdade fornecida pelas entrevistas pode fazer com que os entrevistados não contribuam tanto quanto deveriam. Desta forma, a aplicação do questionário após a realização das entrevistas pode auxiliar o pesquisador a obter maiores informações sobre o conteúdo abordado, sem interferir nas respostas das entrevistas. Além disso, é interessante analisar a implementação dos mecanismos e os resultados obtidos nesta na empresa, o que não era possível com a condução de um estudo de caso. Assim, sugere-se que futuramente seja conduzida uma pesquisa-ação para a avaliação dos resultados.

Os benefícios proporcionados pelo mentoring, apresentado nesta pesquisa como um mecanismo de CC não tecnológico poderão ser analisados com maior profundidade em pesquisas futuras. Podendo levar em consideração o incentivo da organização quanto à sua utilização, e a importância identificada pelos colaboradores que o utilizam. Aplicar a mesma pesquisa em outras empresas do mesmo setor contribuiria para verificar o alinhamento dos resultados obtidos, e identificar as diferenças dos aspectos abordados. Assim, se teria subsídios para realizar um estudo quantitativo, e observar as relações de causa e efeito entre as variáveis. 


\section{REFERÊNCIAS}

ASSOCIAÇÃO BRASILEIRA DE NORMAS TÉCNICAS. NBR ISO 9001:2015: Sistemas de Gestão da Qualidade - Requisitos. Rio de Janeiro, 2015.

BARDIN, L. Análise de Conteúdo. Lisboa: Edições 70, 2009.

BECHKY, B. A. Sharing meaning across occupational communities: The transformation of understanding on a production floor. Organization Science, [S.1.], v. 14, n. 3, p. 312-330. 2003.

BENBYA, H.; BELBALY, N. A. Mechanisms for knowledge management system effectiveness: An exploratory analysis. Knowledge and Process Management, [S.1], v. 12, n. 3, p. 203-216. 2005.

BINNEY, D. The knowledge management spectrum - understanding the KM landscape. Journal of Knowledge Management, [S.1], v. 5, n.1, p. 33-42. 2001.

BOLLINGER, A. S.; SMITH, R. D. Managing organizational knowledge as a strategic asset. Journal of Knowledge Management, [S.1], v. 5, n.1, p. 8-18. 2001.

BOSE, R. Knowledge management metrics. Industrial Management \& Data Systems, [S.1], v. 104, n. 6, p. 457-468. 2004.

CAMPOS, M. Z. C. R.; FERREIRA, M. A. T.; DA SILVA, S. M. Transferência do conhecimento tecnológico gerado em projetos de P\&D no setor elétrico brasileiro: o caso Eletronorte. Revista Economia \& Gestão, v. 9, n. 21, pp. 100-119. 2009.

CHEN, H.; NUNES, M.B.; RAGSDELL, G.; AN, X. Extrinsic and intrinsic motivation for experience grounded tacit knowledge sharing in Chinese software organizations. Journal of Knowledge Management, v. 22, n. 2, p.478-498, 2018.

CURTIS, M.B.; Taylor, E.Z. Developmental mentoring, affective organizational commitment, and knowledge sharing in public accounting firms. Journal of Knowledge Management, v. 22, n. 1, p.142-161, 2018.

DAVENPORT, T. H.; PRUSAK, L. Conhecimento empresarial: como as organizações gerenciam o seu capital intelectual. Rio de Janeiro: Campus, 1998.

DUARTE, E. N. et al. Vantagens do uso de tecnologias pra criação, armazenamento e disseminação do conhecimento em bibliotecas universitárias. Transinformação, Campinas/SP, v. 18, n. 2, p. 131-141. 2006.

FLICK, U. Desenho da Pesquisa Qualitativa. Porto Alegre: Artmed, 2009.

GOMES, F.; OLIVEIRA, M.; CHAVES, M.S. An analysis of the relationship between knowledge sharing and the project management process groups. Knowledge and Process Management. Disponível em: https://doi.org/10.1002/kpm.1578. Acesso em: 16 jul. 2018.

GRACE, T. P. L. Wikis as a knowledge management tool. Journal of Knowledge Management, [S.1], v. 13, n. 4, p. 64-74. 2009.

GUINEA, A. O.; WEBSTER, J.; STAPLES, D. S. A meta-analysis of the consequences on team functioning. Information and Management, v. 49, n. 6, p. 301-308, 2012.

HANSEN, M. T.; NOHRIA, N.; TIERNEY, T. What's your strategy for managing knowledge. Harvard Business Review, [S.1], v. 77, n. 2, p. 106-116. 1999.

HASHEMI, P.; KHADIVAR, A.; SHAMIZANJANI, M. Developing a domain ontology for knowledge management technologies. Online Information Review, v. 42, n. 1, p. 28-44, 2018.

JASIMUDDIN, S. M. Exploring knowledge transfer mechanisms: The case of a UK-based group within a high-tech global corporation. International Journal Of Information Management, [S.1], v. 27, n. 4, p. 294-300. 2007.

JASIMUDDIN, S. M. Storage of transferred knowledge or transfer of stored knowledge: which direction? If both, then how? Proceedings of the Annual Hawaii International 
Conference on System Sciences, $[$ S.1], p. 27. 2005. Disponível em: <http://dx.doi.org/10.1109/HICSS.2005.557>. Acesso em: 24 jun. 2017.

JORGE, C. F. B.; FALÉCO, L. L. A aplicação da gestão do conhecimento como estratégia de competitividade organizacional. Brazilian Journal of Information Studies: Research Trends, [S.1], v. 10, n.3, p. 69-75. 2016.

KANKANHALLI, A. et al. The role of IT in successful knowledge management initiatives. Communications of the ACM, [S.1], v.46, n. 9, p. 69-73. 2003.

LAWRENCE, R. Executive mentoring: turning knowledge into wisdom. Business Strategy Series, [S.1], v. 9, n. 3, p. 126-131. 2008.

MACIEL, A. M. T.; PIMENTEL, R. C. V.; MARCHORI, M. Comunicação e conhecimento: interrelações que permeiam o ambiente organizacional. Revista Digital de Biblioteconomia e Ciência da Informação, Campinas/SP, v.11, n. 2, p. 98-122. 2013.

NONAKA, I.; TAKEUCHI, H. Gestão do Conhecimento. São Paulo: Bookman, 2008.

OLIVEIRA, M.; MAÇADA, A. C. G.; CURADO, C. Adopting Knowledge Management Mechanisms: Evidence from Portuguese Organizations. Knowledge and Process Management, [S.1], v.21, n.4, p.231-245. 2014.

O'SULLIVAN, K. J. Creating and executing an internal communications plan for knowledge management systems deployments. Journal of Knowledge Management, [S.1], v. 11, n. 2, p.102-108. 2007.

SAITO, A.; UMEMOTO, K.; IKEDA, M. A strategy-based ontology of knowledge management technologies. Journal of Knowledge Management, [S.1], v. 11, n. 1, p.97-114. 2007.

SARKA, P.; IPSEN, C. Knowledge sharing via social media in software development: a systematic literature review. Knowledge Management Research \& Practice, v. 15, n.4, p. 594-609, 2017.

SILVA, H. de F. N. et al. As contribuições relativas ao uso de eventos/fóruns para constituição de comunidades de práticas e expressão da inteligência coletica: o caso do bibliocontas. Perspectivas em Ciência da Informação, v. 17, n. 3, p.100-120. 2012.

SILVA, R. H. C. Retração da atividade da construção civil e da indústria trouxe impacto mais acentuado no mercado de trabalho formal das regiões Norte e Sudeste. 06 out. 2016. Disponível em: <https://www.economiaemdia.com.br/ EconomiaEmDia/pdf/ destaque_setorial_05_10_16v3.pdf >. Acesso em: 28 mar. 2017.

SINBORSUL. Balanço Econômico 2015 e Perspectivas 2016. São Leopoldo. Disponível em <http://www.sinborsul.com.br/acervo/1462557230.pdf>. Acesso em: 25 mar. 2017.

SKOK, W.; KALMANOVITCH, C. Evaluating the role and effectiveness of an intranet in facilitating knowledge management: a case study at Surrey County Council. Information \& Management, [S.1], v. 42, n. 5, p.731-744. 2004.

SOMATRI, M.; DJOHAR, A.; ABDULLAH, A.G.; JUHANA, A.; ARYADI, S. Implementation of sharing knowledge management in internship program using web-based information system. Materials Science and Engineering, 288, p.1-10. 2018.

VERONESE, G. S. Métodos para captura de lições aprendidas: Em direção a melhoria continua na gestão de projetos. Revista de Gestão e Projetos, [S.1], v. 5, n. 1, p.71-83. 2014. 